\title{
Representation Theoretical Meaning of the Initial Value Problem for the Toda Lattice Hierarchy II
}

By

\author{
Takashi TAKEBE*
}

\begin{abstract}
The Toda lattice hierarchy is shown to have the virtual Bruhat decomposition of the $A_{\infty}$ group as its parameter space, instead of the Grassmann manifold for the $K P$ hierarchy. The small cells of the Bruhat decomposition are examined. The parametrization of the $K P$ hierarchy is utilized for this purpose with the help of the Miura transformation.
\end{abstract}

\section{§0. Introduction}

This is the continuation of the paper [Take] which shows that solutions of the Toda lattice hierarchy are parametrized by the Bruhat decomposition of the $A_{\infty}$ group in the generic case. The present paper aims to establish this relation in the non generic case. As in the $K P$ theory, where the degenerate cells of the universal Grassmann manifold parametrize pseudo regular solutions, the degenerate cells of the Bruhat decomposition of $A_{\infty}$ parametrize pseudo regular solutions of the Toda lattice hierarchy. (The notion of pseudo regularity will be reviewed in $\$ 1.2$.)

The main problem is how to recover the initial data from non regular solutions. In the generic case, as seen in [Taka] and [Take], the initial data can be recovered just by taking the initial values of the wave functions, but in general this does not work. Hence, we use an indirect method making use of the result of the $K P$ theory. This is enabled by the Miura transformation, which, in this situation, means extracting one component of the flag consisting of the elements of the universal Grassmann manifold.

The term "Bruhat decomposition" is somewhat misleading, because there

Communicated by M. Kashiwara, July 2, 1990.

1991 Mathematics Subject Classification: 17B67, 81R10, 82C23, 58F07, 22E70.

* Department of Mathematics, Faculty of Science, The University of Tokyo, Hongo 7-3-1, Bunkyo-ku, Tokyo, 113, Japan. 
does not exist any group decomposed, but for the moment we retain using this to designate the disjoint union

$$
\coprod_{\sigma} N_{\sigma} \times \sigma H \times N_{+}
$$

Here $N_{+}$(resp. $N_{-}$) is the set of upper (resp. lower) triangular matrices, $H$ is the set of diagonal matrices, $\sigma$ is an element of the "Weyl group" and $N_{\sigma}$ $=N_{-} \cap \sigma N_{-} \sigma^{-1}$. For the "Weyl group" we take the set of charge conserving permutations introduced in $\S 1.1$ instead of the set of finite permutations. This allows us to reduce the whole theory to the periodic system corresponding to the affine Lie algebra $A_{l}^{(1)}$.

The present paper is organized as follows: $\S 1$ is devoted to the preparation, especially in $\S 1.2$ we recall the Sato theory of the $K P$ hierarchy for the non generic case. In $\S 2$ we construct the $\tau$ function and wave functions of the Toda lattice hierarchy, as in the previous paper [Take], in terms of the vacuum expectation value. In $\S 3$, after recalling the Miura transformation in [UT], we show that the pseudo regular wave functions of the Toda lattice hierarchy determine an element of the Bruhat decomposition.

We keep all the notations of [Take].

\section{Acknowledgement}

The present author expresses his great thanks to Professor K. Takasaki for stimulating discussions and to Professors H. Komatsu, Kimio Ueno, and $\mathbf{M}$. Noumi for valuable comments. In particular, it is Professor K. Takasaki who has pointed out the relation between the Miura transformation and the flag of the $K P$ hierarchies.

The author dedicates this paper to the memory of his mother.

\section{§1. Preliminaries}

\section{\$1.1 Charge Conserving Permutations}

As in [Take], a Maya diagram of charge $s$ is a set of strictly decreasing integers $\left\{i_{s}>i_{s-1}>i_{s-2}>\ldots\right\}$ with the condition $i_{k}=k$ for $k \ll 0$, and $\mathscr{M}_{s}$ denotes the set of all Maya diagrams of charge $s$.

Definition 1.1. A permutation $\sigma: \mathbb{Z} \rightarrow \mathbb{Z}$ is called charge conserving, if for all $M \in \mathscr{M}_{s}, s \in \mathbb{Z}$, it satisfies $\sigma(M) \in \mathscr{M}_{s}$. The set of all charge conserving permutations is dnoted as $\Sigma$. We call two charge conserving permutations $\sigma$ and $\sigma^{\prime}$ equal up to finite difference, $\sigma \sim \sigma^{\prime}$, if $\sigma \sigma^{-1}$ is a finite permutation.

Finite permutations and periodic permutations (i.e., $\sigma(k N+r)=k N+\sigma_{N}(r)$ for some $N$, some permutation of $N$ letters $\sigma_{N}$, and all $k \in \mathbb{Z}, r \in \mathbb{Z} / N \mathbb{Z}$ ) are 
examples of charge conserving permutations.

Take one of the equivalence class with respect to $\sim$, and fix its representative $\sigma_{0}$. Then, if $\sigma$ belongs to this equivalence class, we can define its action on the Fock space $\mathscr{F}$ inductively so that it satisfies

$$
\sigma \psi_{i}|M\rangle=\psi_{\sigma(i)} \sigma|M\rangle, \quad \sigma \psi_{i}^{*}|M\rangle=\psi_{\sigma(i)}^{*} \sigma|M\rangle,
$$

for all Maya diagram $M$ and $i \in \mathbf{Z}$, with the normalization

$$
\sigma_{0}|0\rangle=\left|\sigma_{0}\left(\mathbf{Z}_{\leqq 0}\right)\right\rangle \text {. }
$$

Here $\mathbf{Z}_{\leqq 0}$ is the set of all non positive integers and $|0\rangle$ is $\left|\mathbf{Z}_{\leqq 0}\right\rangle$. For finite permutations we take the usual normalization such that

$$
\sigma|M\rangle=\operatorname{det}\left(\left.\sigma\right|_{M}\right)|\sigma(M)\rangle \text {. }
$$

It is easy to see that the dual action on the dual Fock space $\mathscr{F}^{\vee}$ is similarly defined and satisfies

$$
(\langle N| \sigma)|M\rangle=\langle N|(\sigma|M\rangle)=:\langle N|\sigma| M\rangle .
$$

Remark 1.2. With appropriate choice this "action" may be the action of the group of all charge conserving permutations, but we do not need it later.

\section{$\S 1.2$ Pseudo-Regular Solutions of the $K P$ Hierarchy}

Here we recall about the correspondence between solutions of the $K P$ hierarchy and the universal Grassmann manifold, following [SN]. Let $\mathscr{C}$ be an algebra over $\mathbf{C}$ and $\mathscr{R}:=\mathscr{C}[[x]], \mathscr{K}:=\mathscr{C}((x))$. Let $\mathscr{D}_{\mathscr{A}}$ and $\mathscr{E}_{\mathscr{A}}$ be the ring of differential and micro differential operators respectively with coefficients in some ring $\mathscr{A}$. $\partial$ denotes $\partial / \partial_{x}$ as usual. We introduce the decreasing filtration of the ring $\mathscr{E}_{\mathscr{A}}$ by the order of the micro differential operators:

$$
F^{s} \mathscr{E}_{\mathscr{A}}:=\left\{P \in \mathscr{E}_{\mathscr{A}} \mid \operatorname{ord} P \leqq-s\right\} .
$$

Define the infinite dimensional $\mathscr{C}$ linear spaces $\mathscr{V}, V^{\phi}$ and the filtration of $\mathscr{V}$ as follows :

$$
\begin{aligned}
\mathscr{V} & :=\mathscr{E}_{\mathscr{R}} / \mathscr{E}_{\mathscr{R}} x, \quad F^{s} \mathscr{V}:=F^{s} \mathscr{E}_{\mathscr{R}} / \mathscr{E}_{\mathscr{R}} x \cap F^{s} \mathscr{E}_{\mathscr{R}}, \\
V^{\phi} & :=\mathscr{D}_{\mathscr{R}} / \mathscr{E}_{\mathscr{R}} x \cap \mathscr{D}_{\mathscr{R}} .
\end{aligned}
$$

We fix the standard basis of $\mathscr{V}:\left\{e_{i}=\partial^{-i} \bmod \mathscr{E}_{\mathscr{R}} x\right\}_{i \in \mathbf{Z}}$.

Definition 1.3. The universal Grassmann manifold of charge $s \quad U G M^{s}$ is the set of the linear subspaces $U$ of $\mathscr{V}$ such that

$$
\text { index of }\left(U \rightarrow \mathscr{V} / F^{1} \mathscr{V}\right)=s,
$$

that is to say, 


$$
\operatorname{dim}\left(U \cap F^{1} \mathscr{V}\right)=\operatorname{dim}\left(\mathscr{V} /\left(U+F^{1} \mathscr{V}\right)\right)+s<\infty .
$$

The Maya diagram associated to $U$ is

$$
\begin{aligned}
M(U): & =\left\{i \in \mathbf{Z} \mid \operatorname{dim}_{\operatorname{gr}_{i}} U=1\right\} \\
& =\left\{i_{s}>i_{s-1}>i_{s-2}>\cdots\right\} .
\end{aligned}
$$

The canonical frame of $U$ is the basis $\left\{\xi_{k}\right\}_{k \leqq s}$ of $U$ which is determined uniquely by the following conditions: Assume $\xi_{i}=\sum_{j \in \mathbf{Z}} \xi_{j i} e_{j}$. Then

(1) $\xi_{i_{k}, k}=1$.

(2) $\xi_{j k}=0$ if $j<i_{k}$.

(3) $\xi_{i_{k}, l}=0$ if $k>l$.

Before recalling the theory of the $K P$ hierarchy, we are going to recall the correspondence between the universal Grassmann manifold and the pseudo regular micro differential operators.

Definition 1.4. Let

$$
W(x ; \partial)=\partial^{s}+w_{1}(x) \partial^{s-1}+w_{2}(x) \partial^{s-2}+\cdots \in \mathscr{E}_{\mathscr{K}}
$$

be an ordinary micro differential operator of order $s$ with rational coefficients. $W(x ; \partial)$ is called pseudo regular, if there exist non negative integers $m$ and $n$ such that

$$
x^{m} W(x ; \partial), \quad W^{-1}(x ; \partial) x^{n} \in \mathscr{E}_{\mathscr{R}} .
$$

The set of all pseudo regular micro differential operators of order $s$ is denoted as $\mathscr{W}^{s}$.

Theorem 1.5. ([SN] Theorem 9.2) Let $W(x ; \partial)$ be a pseudo regular micro differential operator of order $s$ and fix non positive integers $m$ and $n$ as in Definition 1.4. Then

$$
\left\{\xi \in \mathscr{V} \mid x^{m} W \xi \in V^{\phi}\right\}=W^{-1} x^{n} V^{\phi} \in U G M^{s} .
$$

Defining the element of $U G M^{s} \gamma_{0}(W)$ by the above formula, we obtain the bijection $\gamma_{0}: \mathscr{W}^{s} \rightarrow U G M^{s} . \quad \gamma_{0}(W)$ does not depend on the choice of $m$ and $n$.

Now we are in position of describing the parametrization of the solutions of the $K P$ hierarchy. Set $\mathscr{C}:=\mathbf{C}\left[\left[t_{2}, t_{3}, \ldots\right]\right]$ and identify $x$ with $t_{1}$. Then $\mathscr{R}$ $=\mathbf{C}[[\mathbf{t}]]$ and $\mathscr{K}=\mathbf{C}\left(\left(t_{1}\right)\right)\left[\left[t_{2}, t_{3}, \ldots\right]\right]$, where $\mathfrak{t}=\left(t_{1}, t_{2}, t_{3}, \ldots\right)$.

Definition 1.6. A micro differential operator

$$
W(\mathbb{t} ; \partial)=\partial^{s}+w_{1}(\mathbf{t}) \partial^{s-1}+w_{2}(\mathbb{t}) \partial^{s-2}+\cdots \in \mathscr{E}_{\mathscr{K}}
$$

is called the wave operator of the $K P$ hierarchy, if it satisfies the bilinear residue 
formula:

$$
\oint_{\lambda=\infty} w(\mathbf{t} ; \lambda) w^{*}\left(\mathbf{t}^{\prime} ; \lambda\right) \frac{d \lambda}{2 \pi i \lambda}=0,
$$

for all $\mathbf{t}$ and $\mathbf{t}^{\prime}$, where

$$
\begin{aligned}
& w(\mathbf{t} ; \lambda)=\left(W^{*}\right)^{-1}(\mathbf{t} ; \partial) e^{-\xi(\mathbf{t}, \lambda)}, \quad w^{*}(\mathbf{t} ; \lambda)=\lambda W(\mathbf{t} ; \partial) e^{\xi(\mathbf{t}, \lambda)}, \\
& \xi(\mathbf{t}, \lambda)=\sum_{n=1}^{\infty} t_{n} \lambda^{n} .
\end{aligned}
$$

We call $w(\mathbf{t} ; \lambda)$ the wave function and $w^{*}(\mathbf{t} ; \lambda)$ its dual. $\mathscr{W}_{\boldsymbol{K} \boldsymbol{P}}^{s}$ denotes the set of all wave operators of the $K P$ hierarchy in $\mathscr{W}^{s}$.

Pseudo regularity of the wave operator is also described as follows: there exist non negative integers $m$ and $n$ such that $t_{1}{ }^{m} w(\mathbf{t} ; \lambda)$ and $t_{1}{ }^{n} w^{*}(\mathbf{t} ; \lambda)$ belong to $\mathscr{R}[[\lambda]]$. In this case we call the wave functions pseudo regular.

One of the most remarkable claims of the Sato theory is the following fundamental result.

Theorem 1.6. ([SN] Theorem 12.3) For any pseudo regular micro differential operator

$$
W^{0}(x ; \partial)=\partial^{s}+w_{1}(x) \partial^{s-1}+w_{2}(x) \partial^{s-2}+\cdots,
$$

there exists one and only one pseudo regular wave operator of the KP hierarchy $W(\mathbf{t} ; \partial) \in \mathscr{W}^{s}$ such that $W\left(t_{1}, 0,0, \ldots ; \partial\right)=W^{0}\left(t_{1} ; \partial\right)$. Hence there is the bijective mapping $\gamma: \mathscr{W}_{K P}^{s} \rightarrow U G M^{s}$ which sends $W(\mathbf{t} ; \partial)$ to $\gamma_{0}\left(W\left(t_{1}, 0,0, \ldots ; \partial\right)\right)$.

The so called $\tau$ function of the $K P$ hierarchy is introduced as follows.

Proposition 1.7. ([SN] $\S 13)$ Let $w(\mathbf{t} ; \lambda)$ and $w^{*}(\mathbf{t} ; \lambda)$ be a pseudo regular wave function of the KP hierarchy and its dual. Then there exists $\tau(\mathrm{t}) \in \mathscr{R}$ such that

$$
\begin{aligned}
& w(\mathbf{t} ; \lambda)=e^{-\xi(\mathbf{t}, \lambda)} \tau\left(\mathbf{t}+\epsilon\left(\lambda^{-1}\right)\right) / \tau(\mathbf{t}), \\
& w^{*}(\mathbf{t} ; \lambda)=e^{\xi(\mathbf{t}, \lambda)} \tau\left(\mathbf{t}-\epsilon\left(\lambda^{-1}\right)\right) / \tau(\mathbf{t}),
\end{aligned}
$$

where $\epsilon(\mu):=\left(\mu, \mu^{2} / 2, \mu^{3} / 3, \ldots\right)$.

We can reconstruct the $\tau$ and wave functions of the KP hierarchy from the element of $U G M^{s}$ as follows.

Proposition 1.8. ([DJKM], [KNTY]) Let $U$ be an element of $U G M^{s}$ and $M(U),\left\{\xi_{k}\right\}_{k \leqq s}$ be as in Definition 1.3, and let the sequence $i_{s+1}<i_{s+2}<\cdots$ be the complement of $M(U): M(U) \cup\left\{i_{k}\right\}_{k>s}=\mathbf{Z}$. Let $g$ be the matrix of $\mathbf{Z} \times \mathbf{Z}$ type whose $k$-th column vector is $\xi_{k}$ if $k \leqq s$ and $\left(\delta_{i, i_{k}}\right)$ if $k>s$. Then 


$$
\begin{aligned}
& \tau(\mathbf{t} ; U)=\left\langle s\left|e^{J_{+}(\mathbf{t})} g\right| s\right\rangle, \\
& w(\mathbf{t} ; \lambda ; U)=\left\langle s-1\left|e^{J_{+}(\mathbf{t})} \psi(\lambda) g\right| s\right\rangle, \\
& w^{*}(\mathbf{t} ; \lambda ; U)=\left\langle s+1\left|e^{J_{+}(\mathbf{t})} \psi^{*}(\lambda) g\right| s\right\rangle .
\end{aligned}
$$

(For the definition of $J_{+}$see Definition 2.1.)

\section{§2. Construction of $\tau$ and Wave Functions}

In this section we construct the $\tau$ function and wave functions of the Toda lattice hierarchy from an element of the virtual Bruhat decomposition of the $A_{\infty}$ group. As in [Take] we use the following notations: $A_{\infty}=\mathfrak{n}_{-} \oplus \mathfrak{h} \oplus \mathfrak{n}_{+}$is the triangular decomposition of the infinite rank affine Lie algebra $A_{\infty}$, which acts on the Fock space $\mathscr{F}$, and the action of $\mathfrak{n}_{ \pm}$and $\mathfrak{h}$ can be lifted to that of $N_{ \pm}$ $=\exp n_{ \pm}$and $H=\exp \mathfrak{h}$.

With these terms the $\tau$ function and the wave functions are defined in the same way as in [Take] $\S 2$.

Definition 2.1. Let $g=\left(g_{-}, \sigma, e^{u}, g_{+}\right)$be an element of $N_{-} \times \Sigma \times H$ $\times N_{+}(u \in \mathfrak{h})$. The $\tau$ function is the formal power series of the indeterminants $t$ $=\left(\mathbf{t}_{+}, \mathfrak{t}_{-}\right)=\left(t_{1}, t_{2}, \ldots ; t_{-1}, t_{-2}, \ldots\right)$ defined by

$$
\tau(s ; \mathbf{t} ; g):=\left\langle s\left|\exp \left(J_{+}\left(\mathbf{t}_{+}\right)\right) g_{-} \sigma e^{u} g_{+} \exp \left(-J_{-}\left(\mathbf{t}_{-}\right)\right)\right| s\right\rangle, \quad s \in \mathbb{Z},
$$

where

$$
\begin{array}{ll}
J_{+}\left(\mathbf{t}_{+}\right):=\sum_{n>0} J_{n} t_{n}, & J_{-}\left(\mathbf{t}_{-}\right):=\sum_{n<0} J_{n} t_{n}, \\
J_{n}:=\sum_{j \in \mathbf{Z}}: \psi_{j}^{*} \psi_{j+n}: & n \in \mathbb{Z} .
\end{array}
$$

Note that the product $g_{-} \sigma e^{u} g_{+}$is not defined in general, and that the expression (2.1) nevertheless makes sense. Hereafter we abbreviate $g_{-} \sigma e^{u} g_{+}$as $g$.

Definition 2.2. The wave functions attached to $g$ are the following four series :

$$
\begin{aligned}
& w^{(\infty)}(s ; \mathfrak{t} ; \lambda ; g):=\left\langle s-1\left|e^{J_{+}\left(\mathbf{t}_{+}\right)} \psi(\lambda) g e^{-J_{-}(\mathbf{t}-)}\right| s\right\rangle / \tau(s ; \mathfrak{t} ; g), \\
& w^{(\infty) *}(s ; \mathfrak{t} ; \lambda ; g):=\left\langle s+1\left|e^{J_{+}(\mathbf{t}+)} \psi^{*}(\lambda) g e^{-J_{-}(\mathbf{t}-)}\right| s\right\rangle / \tau(s ; \mathfrak{t} ; g),
\end{aligned}
$$

corresponding to the singularity at $\lambda=\infty$, and

$$
\begin{aligned}
& w^{(0)}(s ; \mathfrak{t} ; \lambda ; g):=\left\langle s-1\left|e^{J_{+}\left(\mathbf{t}_{+}\right)} g \psi(\lambda) e^{-J_{-}(\mathbf{t}-)}\right| s\right\rangle / \tau(s ; \mathfrak{t} ; g), \\
& w^{(0) *}(s ; \mathfrak{t} ; \lambda ; g):=\left\langle s+1\left|e^{J_{+}\left(\mathbf{t}_{+}\right)} g \psi^{*}(\lambda) e^{-J_{-}(\mathbf{t}-)}\right| s\right\rangle / \tau(s ; \mathbf{t} ; g),
\end{aligned}
$$

corresponding to the singularity at $\lambda=0$. Here $\psi^{(*)}(\lambda)$ 's are the fermion field operators 


$$
\psi(\lambda):=\sum_{n \in \mathbf{Z}} \psi_{n} \lambda^{-n}, \quad \psi^{*}(\lambda):=\sum_{n \in \mathbf{Z}} \psi_{n}^{*} \lambda^{n} .
$$

It is easy to see that these wave functions are pseudo regular (with respect to $t_{1}$ (in the case of $w^{(\infty)}$ and $\left.w^{(\infty) *}\right), t_{-1}$ (in the case of $w^{(0)}$ and $w^{(0) *}$ ) respectively) in the sense of $\S 1.2$.

Proposition 2.4 of [Take] holds in this case also: The wave functions are written in terms of the $\tau$ function like

$$
\begin{aligned}
& w^{(\infty)}(s ; \mathbf{t} ; \lambda ; g)=e^{\xi\left(-\mathbf{t}_{+}, \lambda\right)} \lambda^{-s} \tau\left(s ; \mathbf{t}_{+}+\epsilon\left(\lambda^{-1}\right), \mathbf{t}_{-} ; g\right) / \tau(s ; \mathbf{t} ; g), \\
& w^{(\infty) *}(s ; \mathbf{t} ; \lambda ; g)=e^{\xi\left(\mathbf{t}_{+}, \lambda\right)} \lambda^{s+1} \tau\left(s ; \mathbf{t}_{+}-\epsilon\left(\lambda^{-1}\right), t_{-} ; g\right) / \tau(s, \mathbf{t} ; g), \\
& w^{(0)}(s ; \mathbf{t} ; \lambda ; g)=e^{\xi\left(-\mathbf{t}_{-}, \lambda^{-1}\right)} \lambda^{-s} \tau\left(s-1 ; \mathbf{t}_{+}, \mathbf{t}_{-}+\epsilon(\lambda) ; g\right) / \tau(s ; \mathbf{t} ; g), \\
& w^{(0) *}(s ; \mathbf{t} ; \lambda ; g)=e^{\xi\left(\mathbf{t}_{-}, \lambda^{-1}\right)} \lambda^{s+1} \tau\left(s+1 ; \mathbf{t}_{+}, \mathbf{t}_{-}-\epsilon(\lambda) ; g\right) / \tau(s ; \mathbf{t} ; g) .
\end{aligned}
$$

The main claim of this section is the following.

Proposition 2.3. The wave functions defined in Definition 2.2 satisfy the bilinear relation of the Toda lattice hierarchy. i.e.,

$$
\begin{aligned}
& \oint_{\infty} \frac{d \lambda}{2 \pi i \lambda} w^{(\infty)}(s ; \mathbf{t} ; \lambda ; g) w^{(\infty) *}\left(s^{\prime} ; \mathbf{t}^{\prime} ; \lambda ; g\right) \\
& \quad+\oint_{0} \frac{d \lambda}{2 \pi i \lambda} w^{(0)}(s ; \mathbf{t} ; \lambda ; g) w^{(0) *}\left(s^{\prime} ; \mathbf{t}^{\prime} ; \lambda ; g\right)=0
\end{aligned}
$$

for all $s, s^{\prime}, \mathbf{t}, \mathbf{t}^{\prime}$.

Proof. As in Proposition 2.5 of [Take], the above bilinear equation is equivalent to

$$
\sum_{i \in \mathbf{Z}}\left\langle M\left|\psi_{i} g\right| N\right\rangle\left\langle M^{\prime}\left|\psi_{i}^{*} g\right| N^{\prime}\right\rangle=\sum_{i \in \mathbf{Z}}\left\langle M\left|g \psi_{i}\right| N\right\rangle\left\langle M^{\prime}\left|g \psi_{i}^{*}\right| N^{\prime}\right\rangle,
$$

for any Maya diagrams $M \in \mathscr{M}_{s-1}, N \in \mathscr{M}_{s}, M^{\prime} \in \mathscr{M}_{s^{\prime}+1}, N^{\prime} \in \mathscr{M}_{s^{\prime}}$. By (1.1), (1.2) and (1.3) of [Take], the proof of this equation is reduced to the cofactor expansion (Lemma 2.6 [Take]) and the identity

$$
\left\langle M\left|\sigma \psi_{i}\right| N\right\rangle=\left\langle M\left|\psi_{\sigma(i)} \sigma\right| N\right\rangle, \quad M \in \mathscr{M}_{s}, N \in \mathscr{M}_{s+1},
$$

which follows immediately from (1.1).

\section{§3. Recovery of the Initial Data}

\section{§3.1 Miura Transformation and the Flag}

Here we recall about the Miura transformation in order to utilize the information about the $K P$ hierarchy for the parametrization of the solution of 
the Toda lattice hierarchy. In this context the Miura transformation means extracting one component of a flag consisting of elements of the universal Grassmann manifold.

Definition 3.1. A set of pseudo regular wave functions of Toda lattice hierarchy is the quaternary of formal power series $w=\left(w^{(\infty)}, w^{(\infty) *}, w^{(0)}, w^{(0) *}\right)$ satisfying:

(i) They are of the following form:

$$
\begin{aligned}
& w^{(\infty)}(s ; \mathfrak{t} ; \lambda)=\hat{w}^{(\infty)}(s ; \mathfrak{t} ; \lambda) \lambda^{-s} e^{-\xi\left(\mathbf{t}_{+}, \lambda\right)}, \\
& w^{(\infty) *}(s ; \mathfrak{t} ; \lambda)=\hat{w}^{(\infty) *}(s ; \mathfrak{t} ; \lambda) \lambda^{s+1} e^{\xi\left(\mathbf{t}_{+}, \lambda\right)},
\end{aligned}
$$

corresponding to the singularity $\lambda=\infty$, and

$$
\begin{aligned}
& w^{(0)}(s ; \mathbb{t} ; \lambda)=\hat{w}^{(0)}(s ; \mathfrak{t} ; \lambda) \lambda^{-s} e^{-\xi\left(\mathbf{t}_{-}, \lambda^{-1}\right)}, \\
& w^{(0) *}(s ; \mathbf{t} ; \lambda)=\hat{w}^{(0) *}(s ; \mathbf{t} ; \lambda) \lambda^{s+1} e^{\xi\left(\mathbf{t}_{-}, \lambda^{-1}\right)},
\end{aligned}
$$

corresponding the singularity $\lambda=0$, where

$$
\begin{aligned}
& \hat{w}^{\left(\begin{array}{c}
0 \\
\infty
\end{array}\right)}(s ; \mathfrak{t} ; \lambda)=\sum_{j=0}^{\infty} \hat{w}^{\left(\begin{array}{c}
0 \\
\infty
\end{array}\right)}(s ; \mathfrak{t}) \lambda^{ \pm j}, \\
& \hat{w}^{\left(\begin{array}{c}
0 \\
\infty
\end{array}\right) *}(s ; \mathfrak{t} ; \lambda)=\sum_{j=0}^{\infty} \hat{w}_{\infty}^{(0) *}(s ; \mathfrak{t}) \lambda^{ \pm j} .
\end{aligned}
$$

(ii) $\hat{w}^{(\infty)}{ }_{j}(s ; \mathbf{t}), \hat{w}^{(\infty) *}{ }_{j}(s ; \mathbf{t})$ belong to $\mathbf{C}\left(\left(t_{1}\right)\right)\left[\left[t_{2}, t_{3}, \ldots ; \mathbf{t}_{-}\right]\right]$, and $\hat{w}^{(\infty)}{ }_{0}=\hat{w}^{(\infty) *}{ }_{0}$ $=1$. Similarly $\hat{w}^{(0)}{ }_{j}(s ; \mathbf{t}), \hat{w}^{(0)}{ }_{j}(s ; \mathbf{t})$ belong to $\mathbf{C}\left(\left(t_{-1}\right)\right)\left[\left[\mathfrak{t}_{+} ; t_{-2}, t_{-3}, \ldots\right]\right]$, and the Laurent series expansions of $\hat{w}^{(0)}{ }_{0}$ and $\hat{w}^{(0)} *_{0}$ with respect to $t_{-1}$ are

$$
\begin{aligned}
& \hat{w}^{(0)}{ }_{0}(s ; \mathfrak{t})=t_{-1}^{k_{s}}+o\left(t_{-1}^{k_{s}}\right), \\
& \hat{w}^{(0) *}(s ; \mathfrak{t})=t_{-1}^{-k_{s}}+o\left(t_{-1}^{-k_{s}}\right) .
\end{aligned}
$$

(iii) For each $s$ there exists a sufficient large non negative integer $m=m(s)$, such that

$$
\begin{aligned}
& t_{1}^{m} \hat{w}^{(\infty)}(s ; \mathbb{t} ; \lambda), t_{1}^{m} \hat{w}^{(\infty) *}(s ; \mathbf{t} ; \lambda) \in \mathbb{C}[[\mathbf{t}]]\left[\left[\lambda^{-1}\right]\right], \\
& t_{-1}^{m} \hat{w}^{(0)}(s ; \mathfrak{t} ; \lambda), t_{-1}^{m} \hat{w}^{(0) *}(s ; \mathfrak{t} ; \lambda) \in \mathbb{C}[[\mathbf{t}]][[\lambda]] .
\end{aligned}
$$

(iv) (The bilinear residue formula)

$$
\begin{aligned}
& \oint_{\infty} \frac{d \lambda}{2 \pi i \lambda} w^{(\infty)}(s ; \mathfrak{t} ; \lambda ; g) w^{(\infty) *}\left(s^{\prime} ; \mathfrak{t}^{\prime} ; \lambda ; g\right) \\
& \quad+\oint_{0} \frac{d \lambda}{2 \pi i \lambda} w^{(0)}(s ; \mathbf{t} ; \lambda ; g) w^{(0) *}\left(s^{\prime} ; \mathbf{t}^{\prime} ; \lambda ; g\right)=0
\end{aligned}
$$

for all $s, s^{\prime}, \mathfrak{t}, \mathfrak{t}^{\prime}$. 
Remark 3.2. The wave functions defined above are slightly different from those defined in [UT] (1.2.19).

Putting $\mathbf{t}_{-}=\mathbf{t}^{\prime}-=0$ and $s=s^{\prime}$ in (iv) of Definition 3.1, we obtain

$$
\oint_{\lambda=\infty} w^{(\infty)}\left(s ; \mathbf{t}_{+}, 0 ; \lambda\right) w^{(\infty) *}\left(s ; \mathbf{t}^{\prime}{ }_{+}, 0 ; \lambda\right) \frac{d \lambda}{2 \pi i \lambda}=0,
$$

which is the residue formula of the $K P$ hierarchy (cf. Definition 1.6). Therefore $W\left(s ; \mathbf{t}_{+} ; \partial\right):=\hat{w}^{(\infty) *}\left(s ; \mathbf{t}_{+}, 0 ; \partial\right) \partial^{s}$ is a pseudo regular wave operator of the KP hierarchy with respect to $\mathbf{t}_{+}$. Moreover these wave operators are related to each other. Namely

Proposition 3.3. ([UT] p. 14) Set

$$
b_{0}(s):=\hat{w}_{1}^{(\infty)}(s+1)+\hat{w}^{(\infty) *}{ }_{1}(s) .
$$

Then

$$
\left(\partial_{t_{1}}-b_{0}(s)\right) w^{(\infty) *}(s ; \mathbf{t} ; \lambda)=w^{(\infty) *}(s+1 ; \mathbf{t} ; \lambda) .
$$

Hence

$$
\left(\partial_{t_{1}}-b_{0}(s)\right) W(s ; \mathbf{t} ; \partial)=W(s+1 ; \mathbf{t} ; \partial)
$$

Corollary 3.4. From a set of wave functions of the Toda lattice hierarchy $w$ $=\left(w^{(\infty)}, w^{(\infty) *}, w^{(0)}, w^{(0) *}\right)$ we get a flag

$$
\begin{aligned}
\gamma(w) & :=\left\{U_{s}=\gamma(W(s ; \mathbf{t} ; \partial))\right\}_{s \in \mathbf{Z}}, \\
& \cdots \subset U_{s} \subset U_{s+1} \subset \cdots, \\
& \operatorname{dim}\left(U_{s+1} / U_{s}\right)=1 .
\end{aligned}
$$

Proof. For each $s \in \mathbf{Z}$, we fix a non negative integer $m(s)$ as in Definition 3.1 (iii). Fix $s$ and $\xi \in U_{s}$, and set $m:=2 m(s)+m(s+1)+1$. Then, by definition, $\xi$ satisfies

$$
t_{1}^{m(s)} W\left(s ; t_{1}, 0,0, \ldots ; \partial\right) \xi \in V^{\phi} .
$$

Now Proposition 3.3 implies

$$
\begin{gathered}
t_{1}^{m} W\left(s+1 ; t_{1}, 0,0, \ldots ; \partial\right) \xi=t_{1}^{m}\left(\partial-b_{0}(s)\right) W\left(s ; t_{1}, 0,0, \ldots ; \partial\right) \xi \\
\in t_{1}^{m}\left(\partial-b_{0}(s)\right) t_{1}^{-m(s)} V^{\phi} .
\end{gathered}
$$

Since $t_{1}^{m}\left(\partial-b_{0}(s)\right) t_{1}^{-m(s)}$ belongs to $\mathscr{D}_{\mathscr{R}}$, the right hand side of the above equation is contained in $V^{\phi}$. This means $U_{s} \subset U_{s+1}$. As $U_{s} \in U G M^{s}$, the last statement of the corollary is obvious.

The Miura transformation means originally a transformation which relates the 
solutions of the $K d V$ equation, the $M K d V$ equation (and the Sine-Gordon equation) with each other. Therefore we may well call the projection from the flag to the universal Grassmann manifold $\gamma(w) \mapsto \gamma(W(s ; \mathbf{t} ; \partial))$ the Miura transformation.

Remark 3.5. It is easy to see that $\left(w^{(0)}(s+1 ; \mathbf{t} ; \lambda) / \hat{w}^{(0)}{ }_{0}(s+1 ; \mathbf{t}), w^{(0) *}(s\right.$ $\left.-1 ; \mathbf{t} ; \lambda) / \hat{w}^{(0) *}{ }_{0}(s-1 ; \mathbf{t})\right)$ is also a pair of the pseudo regular wave functions of the $K P$ hierarchy.

\section{\$3.2 Reconstruction of the Initial Data}

Let $w=\left(w^{(\infty)}, w^{(\infty) *}, w^{(0)}, w^{(0) *}\right)$ be a set of the pseudo regular wave functions of the Toda lattice hierarchy, and $\tau$ be the corresponding $\tau$ function which is determined by $w$ up to the multiplicative constant as (2.2). In this section we reconstruct from $w$ and $\tau$ the data $g=\left(g_{-}, \sigma, e^{u}, g_{+}\right)$which belong to $N_{-} \times \Sigma \times H \times N_{+}(u \in \mathfrak{h})$ and are related to $w$ and $\tau$ as in Definition 2.1 and 2.2.

First we fix $\mathbf{t}_{-}=0$ and recover the data $\left(g_{-}, \sigma, e^{u}\right)$. As seen in $\S 3.1$, there exists a flag $\gamma(w)=\left\{U_{s}\right\}_{s \in \mathbf{Z}}$, such that every component $U_{s}$ corresponds to the wave function of the KP hierarchy $w^{(\infty)}\left(s ; \mathbb{t}_{+}, \mathbf{t}_{-}=0 ; \lambda\right), w^{(\infty) *}\left(s ; \mathbb{t}_{+}, \mathbb{t}_{-}\right.$ $=0 ; \lambda)$. We fix the base of $\mathscr{V}\left\{\xi_{k}\right\}_{k \in \mathbf{Z}}$ as follows:

(i) $\left\{\xi_{k}\right\}_{k \leqq s}$ is a base of $U_{s}$.

(ii) Let $\left\{\xi_{k}^{\prime}\right\}_{k \leqq s-1}$ be the canonical frame of $U_{s-1}$ (Definition 1.3). Then $\left\{\xi_{k}^{\prime}\right\}_{k \leqq s-1} \cup\left\{\xi_{s}\right\}$ is the canonical frame of $U_{s}$ up to the order.

The existence is obvious. Condition (ii) of the definition of the canonical frame yields that there exists the charge conserving permutation $\sigma: \mathbb{Z} \rightarrow \mathbb{Z}$ such that the matrix

$$
g_{-}:=\left(\ldots, \xi_{\sigma(k)}, \xi_{\sigma(k+1)}, \ldots\right)
$$

is lower triangular with diagonal elements 1 (i.e. $g_{-} \in N_{-}$). Here $\xi_{k}$ is identified with the column vector by means of the base $\left\{e_{i}\right\}_{i \in \mathbf{Z}}$ of $\mathscr{V}$.

When we identify $\sigma$ with its matrix representation $\left(\delta_{i \sigma(j)}\right)_{i, j \in \mathbf{Z}}$,

$$
g_{-} \sigma=\left(\ldots, \xi_{k}, \xi_{k+1}, \ldots\right) \text {. }
$$

In view of Proposition 1.8 this leads to

$$
\begin{aligned}
& \tau\left(s ; \mathbf{t}_{+}, 0\right)=e^{v_{s}}\left\langle s\left|e^{J_{+}\left(\mathbf{t}_{+}\right)} g_{-} \sigma\right| s\right\rangle, \\
& w^{(\infty)}\left(s ; \mathbf{t}_{+}, 0 ; \lambda\right)=e^{v_{s}}\left\langle s-1\left|e^{J_{+}\left(\mathbf{t}_{+}\right)} \psi(\lambda) g_{-} \sigma\right| s\right\rangle / \tau\left(s ; \mathbf{t}_{+}, 0\right), \\
& w^{(\infty) *}\left(s ; \mathbf{t}_{+}, 0 ; \lambda\right)=e^{v_{s}}\left\langle s+1\left|e^{J_{+}\left(\mathbf{t}_{+}\right)} \psi^{*}(\lambda) g_{-} \sigma\right| s\right\rangle / \tau\left(s ; \mathbf{t}_{+}, 0\right) .
\end{aligned}
$$

The factor $e^{v_{s}}$ arises from the multiplicative ambiguity of the $\tau$ function of the 
$K P$ hierarchy. It is easy to see that there exists $u \in \mathfrak{h}$ such that $e^{u}|s\rangle$ $=e^{v_{s}}|s\rangle$. Using this, we can write

$$
\tau\left(s ; \mathbf{t}_{+}, 0\right)=\left\langle s\left|e^{J_{+}(\mathbf{t}+)} g_{-} \sigma e^{u}\right| s\right\rangle,
$$

and similar equations for $w^{(\infty)}, w^{(\infty) *}$ hold.

So far we have constructed the data $\left(g_{-}, \sigma, e^{u}\right)$. In order to know the remaining datum $g_{+}$we need the regularity property of the $\tau$ function.

Lemma 3.6. The $\tau$ function for the pseudo regular wave function of the Toda lattice hierarchy belongs to $\mathbf{C}[[t]]$.

Proof. The proof of the Theorem 1.7 of [UT] tells that the $\tau$ function is the solution of the consistent system

$$
\begin{aligned}
& \log \hat{w}^{(\infty)}(s ; \mathbf{t} ; \lambda)=\left(\exp \left(\xi\left(\tilde{\partial}_{+}, \lambda^{-1}\right)\right)-1\right) \log \tau(s ; \mathbf{t}), \\
& \log \frac{\hat{w}^{(0)}(s+1 ; \mathbf{t} ; \lambda)}{\hat{w}^{(0)}{ }_{0}(s+1 ; \mathbf{t} ; \lambda)}=\left(\exp \left(\xi\left(\tilde{\partial}_{-}, \lambda\right)\right)-1\right) \log \tau(s ; \mathbf{t}), \\
& \log \hat{w}^{(0)}{ }_{0}(s+1 ; \mathbf{t})=\log \tau(s ; \mathbf{t})-\log \tau(s+1 ; \mathbf{t}),
\end{aligned}
$$

where $\tilde{\partial}_{ \pm}=\left(\partial_{t_{ \pm 1}}, \partial_{t_{ \pm 2}} / 2, \partial_{t_{ \pm 3}} / 3, \ldots\right)$. Each of the first two equations says that $\tau$ is the $\tau$ function of the $K P$ hierarchy with respect to $\mathbf{t}_{+}$and $\mathbf{t}_{-}$ respectively. Because $w^{(\infty)}$ and $w^{(0)} / w^{(0)}$ are pseudo regular wave functions of the KP hierarchy (cf. $\S 3.1$ ), Proposition 1.7 assures the regularity of $\tau$.

Since the Schur functions $\left\{\chi_{Y}\left(\mathbf{t}_{+}\right) \chi_{Y^{\prime}}\left(\mathbf{t}_{-}\right)\right\}_{Y_{,} \boldsymbol{Y}^{\prime}}$ form the basis of $\mathbf{C}[\mathbf{t}], \tau$ can be expanded as follows:

$$
\tau(S ; \mathbf{t})=\sum_{M, N \in M_{s}} \chi_{Y_{M}}\left(\mathbf{t}_{+}\right) \chi_{Y_{N}}\left(\mathbf{t}_{-}\right) a_{N}^{M},
$$

for all $s \in \mathbf{Z}$. Here $Y_{M}$ denotes the Young diagram associated to the Maya diagram $M$. Substituting (3.2) and (2.2) in the bilinear residue formula (Definition 3.1) and using formulae like

$$
\begin{aligned}
\chi_{Y_{M}}\left(\mathbf{t}_{+}-\epsilon\left(\lambda^{-1}\right)\right) & =\left\langle 0\left|e^{J_{+}\left(\mathbf{t}_{+}\right)} \psi\left(\lambda^{-1}\right)\right| M\right\rangle \\
& =\sum_{i \in \mathbf{Z}} \lambda^{i} \epsilon(M, i) \chi_{Y_{M \backslash\{i\}}}\left(\mathbf{t}_{+}\right),
\end{aligned}
$$

(See $\S 1.2$ of [Take] for the meaning of the notations.) we obtain another form of the bilinear relation

$$
\sum_{i \in \mathbf{Z}} \bar{\epsilon}(M, i) \epsilon\left(M^{\prime}, i\right) a_{N}^{M \cup\{i\}} a_{N^{\prime}}^{M^{\prime}\{i\}}=\sum_{i \in \mathbf{Z}} \epsilon(N, i) \bar{\epsilon}\left(N^{\prime}, i\right) a_{N \backslash\{i\}}^{M} a_{N^{\prime} \cup\{i\}}^{M^{\prime}},
$$

for any Maya diagrams $M \in \mathscr{M}_{s-1}, N \in \mathscr{M}_{s}, M^{\prime} \in \mathscr{M}_{s^{\prime}+1}, N^{\prime} \in \mathscr{M}_{s^{\prime}}$.

Setting $\mathbf{t}_{-}=0$, we have from (3.2) 


$$
\tau\left(s ; \mathbf{t}_{+}, 0\right)=\sum_{M \in M_{s}} \chi_{M}\left(\mathbf{t}_{+}\right) a_{\mathrm{vac}(s)}^{M}
$$

where $\operatorname{vac}(s)=\mathbb{Z}_{\leqq s}$, and comparing this with (3.1),

$$
a_{\mathrm{vac}(\mathrm{s})}^{M}=\left\langle M\left|g_{-} \sigma e^{u}\right| s\right\rangle .
$$

In particular,

$$
a_{\mathrm{vac}(s)}^{M\left(U_{s}\right)}=e^{v_{s}} \neq 0
$$

Set

$$
\tau^{\sigma}(s ; \mathbf{t}):=\sum_{M, N \in M_{s}} \chi_{Y_{M}}\left(\mathbf{t}_{+}\right) \chi_{Y_{N}}\left(\mathbf{t}_{-}\right) a_{N}^{\sigma^{-1} M} .
$$

Then (3.3) implies that $\tau^{\sigma}$ is automatically the $\tau$ function of Toda lattice hierarchy and due to $(3.4), \tau(s ; 0) \neq 0$. Hence the wave functions $w^{\left(\begin{array}{l}0 \\ \infty\end{array}\right) \sigma}, w^{\left(\begin{array}{c}0 \\ \infty\end{array}\right) * \sigma}$ constructed from $\tau^{\sigma}$ are regular $(\epsilon \mathbf{C}[[\mathbf{t}]])$. Therefore, as is shown in [Take], there exists $\left(g_{-}^{\prime}, e^{u^{\prime}}, g_{+}\right) \in N_{-} \times H \times N_{+}$such that

$$
\tau^{\sigma}(s ; \mathbf{t}):=\left\langle s\left|\exp \left(J_{+}\left(\mathbf{t}_{+}\right)\right) g_{-}^{\prime} e^{u^{\prime}} g_{+} \exp \left(-J_{-}\left(\mathbf{t}_{-}\right)\right)\right| s\right\rangle, \quad s \in \mathbf{Z} .
$$

This together with (3.3) and (3.5) yields

$$
\tau(s ; \mathfrak{t}):=\left\langle s\left|\exp \left(J_{+}\left(\mathbb{t}_{+}\right)\right) g_{-} \sigma e^{u} g_{+} \exp \left(-J_{-}\left(\mathbf{t}_{-}\right)\right)\right| s\right\rangle, \quad s \in \mathbb{Z} .
$$

Now we obtain the data $\left(g_{-}, \sigma, e^{u}, g_{+}\right)$at last.

Remark 3.7. Setting $\mathbf{t}_{-}=0$ in (3.6) and comparing this with (3.1), we easily see that

$$
g_{-}^{\prime}=\sigma^{-1} g_{-} \sigma, \quad e^{u^{\prime}}=e^{u} .
$$

In fact, owing to the condition (iii) of the definition of the canonical frame ( $\$ 3.1$ ) and the condition (ii) imposed on $\left\{\xi_{k}\right\}, \sigma^{-1} g_{-} \sigma$ also belongs to $N_{-}$, i.e., $g_{-} \in N_{\sigma}$.

\section{§4. Concluding Remarks}

Thus we have obtained bijective mappings

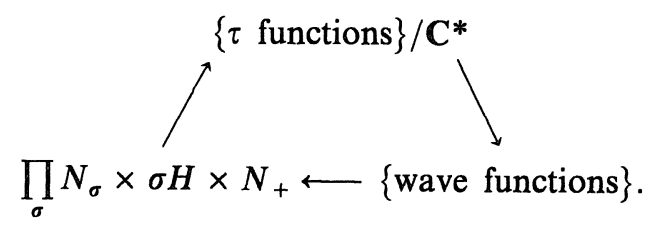

Here $\nearrow$ is Definition 2.1, $\searrow$ is $(2.2)$ and $\leftarrow$ is what we have done in $\S 3.2$.

We end this paper with additional comments. 
1. The Toda lattice hierarchy is known to be embedded in the 2 component $K P$ hierarchy ([UT]). So the relation between the parametrization of the Toda lattice hierarchy which we have constructed in this paper and that of the 2 component $K P$ hierarchy should be further investigated.

2. It is expected that the Toda lattice hierarchy associated to the other type of affine Lie algebra (e.g., $B_{\infty}, C_{\infty}$, and so on) has also the similar parametrization by the Bruhat decomposition.

3. The reconstruction of the data $\left(g_{-}, \sigma, e^{u}, g_{+}\right)$in $\S 3.2$ makes use of the Miura transformation and resorts to the Sato theory of the KP hierarchy. But as the $\tau$ function contains all the informations about the solution, there must be a (possibly combinatorial) method by which those data are obtained directly from the coefficients of the $\tau$ function $a_{N}^{M}$ 's (3.2).

\section{References}

[DJKM] Date, E., Jimbo, M., Kashiwara, M. and Miwa, T., Transformation groups for solition equations, Proceedings of RIMS Symp. Nonlinear integrable systems-classical theory and quantum theory (Kyoto 1981), (1983), 39-119.

[JM] Jimbo, M. and Miwa, T., Solitons and Infinite Dimensional Lie Algebras, Publ. RIMS, Kyoto Univ., 19 (1983), 943-1001.

[KNTY] Kawamoto, N., Namikawa, Y., Tsuchiya, A. and Yamada, Y., Geometric realization of conformal field theory, Commun. Math. Phys., 116 (1988), 247-308.

[SN] Sato, M. and Noumi, M., Soliton equation and universal Grassmann manifold, Sophia University Kokyuroku in Math., 18 (1984). in Japanese.

[Taka] Takasaki, K., Initial value problem for the Toda lattice hierarchy, Adv. Stud. in Pure Math. 4 Group Representation and Systems of Differential Equations (1984), 139-163.

[Take] Takebe, T., Representation theoretical meaning of the initial value problem for the Toda lattice hierarchy I, Lett. Math. Phys. 21 (1991), 77-84.

[UT] Ueno, K. and Takasaki, K., Toda Lattice Hierarchy, Adv. Stud. in Pure Math. 4 Group Representation and Systems of Differential Equations (1984), 1-95. 
